\title{
One-Dimensional Dispersive Magnon Excitation in the Frustrated Spin-2 Chain System $\mathrm{Ca}_{3} \mathrm{Co}_{2} \mathrm{O}_{6}$
}

\author{
Anil Jain, ${ }^{1,2, *}$ P. Y. Portnichenko, ${ }^{3}$ Hoyoung Jang, ${ }^{1,4}$ G. Jackeli, ${ }^{1}$ G. Friemel, ${ }^{1}$ \\ A. Ivanov, ${ }^{5}$ A. Piovano, ${ }^{5}$ S. M. Yusuf, ${ }^{2}$ B. Keimer, ${ }^{1}$ and D. S. Inosov ${ }^{3,1, \dagger}$ \\ ${ }^{1}$ Max-Planck-Institut für Festkörperforschung, Heisenbergstraße 1, D-70569 Stuttgart, Germany \\ ${ }^{2}$ Solid State Physics Division, Bhabha Atomic Research Centre, Mumbai 400085, India \\ ${ }^{3}$ Institut für Festkörperphysik, TU Dresden, D-01069 Dresden, Germany \\ ${ }^{4}$ Stanford Synchrotron Radiation Lightsource, SLAC National Accelerator Laboratory, Menlo Park, California 94025, USA \\ ${ }^{5}$ Institut Laue-Langevin, 6 rue Jules Horowitz, F-38042 Grenoble Cedex 9, France
}

\begin{abstract}
Using inelastic neutron scattering, we have observed a quasi-one-dimensional dispersive magnetic excitation in the frustrated triangular-lattice spin-2 chain oxide $\mathrm{Ca}_{3} \mathrm{Co}_{2} \mathrm{O}_{6}$. At the lowest temperature $(T=1.5 \mathrm{~K})$, this magnon is characterized by a large zone-center spin gap of $\sim 27 \mathrm{meV}$, which we attribute to the large single-ion anisotropy, and disperses along the chain direction with a bandwidth of $\sim 3.5 \mathrm{meV}$. In the directions orthogonal to the chains, no measurable dispersion was found. With increasing temperature, the magnon dispersion shifts towards lower energies, yet persists up to at least $150 \mathrm{~K}$, indicating that the ferromagnetic intrachain correlations survive up to 6 times higher temperatures than the long-range interchain antiferromagnetic order. The magnon dispersion can be well described within the predictions of linear spin-wave theory for a system of weakly coupled ferromagnetic chains with large single-ion anisotropy, enabling the direct quantitative determination of the magnetic exchange and anisotropy parameters.
\end{abstract}

PACS numbers: 75.30.Ds 75.50.Ee 78.70.Nx 75.10.Pq

Frustrated antiferromagnets attract much theoretical and experimental attention because of their peculiar magnetic properties. The geometry of the underlying lattice or competing interactions in these systems may give rise to a macroscopic ground-state degeneracy and thus can prevent the onset of a long-range magnetic ordering (LRO) down to the absolute zero temperature, $T=0 \mathrm{~K}^{1,2}$ Due to the large ground-state degeneracy, small perturbations, such as further-neighbor interaction, single-ion anisotropy, spinlattice interactions, or magnetic field, can give rise to a variety of magnetically ordered states. ${ }^{3}$ Quasi-two-dimensional (2D) triangular-lattice antiferromagnets (TLAF) have been extensively studied as exemplar frustrated spin systems. ${ }^{4-8}$ Their magnetic phase diagrams strongly depend on the exchange anisotropy. A typical 2D Heisenberg TLAF with nearest-neighbor interactions orders in the noncollinear " $120^{\circ}$ structure" even in the extreme quantum spin- $\frac{1}{2}$ case. ${ }^{9}$ However, the 2D Ising TLAF is known to display no LRO down to $T=0 \mathrm{~K}^{10}$ So far, experimental realizations of the Ising TLAF are restricted to a few systems, where ferromagnetic $(\mathrm{FM})^{11-13}$ or antiferromagnetic $(\mathrm{AFM})^{14}$ spin chains are arranged on a triangular lattice in the plane perpendicular to the chains. Among them, compounds with FM chains offer a rich playground to investigate a variety of exotic magnetic phases cooperatively induced by the dimensionality reduction, magnetic anisotropy, geometrical frustration, and magnetic field. ${ }^{11,12}$

As a model system of the Ising TLAF, $\mathrm{Ca}_{3} \mathrm{Co}_{2} \mathrm{O}_{6}$ exhibits many intriguing properties, such as field-induced magnetization steps, ${ }^{15-19}$ time-dependent magnetic order, ${ }^{20,21}$ magnetodielectric coupling ${ }^{22}$ and others. ${ }^{23-28}$ It has a rhombohedral structure (space group $R \overline{3} c$ ) with a hexagonal arrangement of one-dimensional (1D) chains consisting of alternating face-sharing $\mathrm{CoO}_{6}$ octahedra (OCT) and $\mathrm{CoO}_{6}$ trigonal prisms (TP) with respectively low-spin $(S=0)$ and high-spin $(S=2)$ states of the $\mathrm{Co}^{3+}$ ions. ${ }^{26,29}$ Besides, the large single-ion anisotropy of the $\mathrm{Co}_{\mathrm{TP}}^{3+}$ ions leads to an Ising character of their spins, pointing along the $c$-axis, while strong FM intrachain and weaker AFM interchain interactions combined with a triangular-lattice arrangement of spin chains give rise to a geometric frustration. ${ }^{30}$

An effective 2D Ising model was initially proposed ${ }^{31,32}$ to explain the dc magnetization ${ }^{15}$ and neutron-diffraction ${ }^{33-35}$ data. In this "rigid chain" model, every chain was replaced by a fictitious classical Ising spin. However, the recently observed incommensurability of the magnetic structure below the Néel temperature, $T_{\mathrm{N}} \approx 24 \mathrm{~K},{ }^{36,37}$ and the timedependent magnetic order-order transition from the incommensurate to the commensurate half-integer AFM structure below $\sim 10 \mathrm{~K}^{21}$ have challenged the validity of this model. Consequently, a 3D lattice spin model was proposed, ${ }^{38,39}$ in which the nearest- and next-nearest-neighbor interchain AFM interactions were assumed to compete with the dominant FM intrachain coupling.

While $\mathrm{Ca}_{3} \mathrm{Co}_{2} \mathrm{O}_{6}$ has been extensively studied using various experimental probes, the small size of the available single crystals has so far precluded measurements of magnetic excitations in this compound by inelastic neutron scattering (INS), which could enable a quantitative estimate of the exchange couplings and single-ion anisotropy. In this Letter, we use the INS technique to directly probe the magnetic excitations in $\mathrm{Ca}_{3} \mathrm{Co}_{2} \mathrm{O}_{6}$. For these measurements, we meticulously coaligned $\sim 400 \mathrm{mg}$ of small needle-shaped crystals into a mosaic on an $\mathrm{Al}$ plate. The c-axis mosaicity of the aligned crystal assembly was $\lesssim 3.0^{\circ}$. The AFM transition at $\sim 24 \mathrm{~K}$ has been confirmed by magnetic susceptibility ${ }^{40}$ and neutron diffraction, in agreement with earlier results. ${ }^{41,42}$ We performed the INS measurements at the thermal-neutron triple-axis spectrometer IN8 (ILL, Grenoble, France), which was operated in the Flatcone multi-analyzer configuration. ${ }^{43}$ The sample was mounted with its [1 $\overline{1} 0]$ and [001] directions in the horizontal scattering plane.

In Fig. $1(\mathrm{a}-\mathrm{c})$, we show constant-energy maps of the inelastic intensity distribution along the $(H \bar{H} L)$ plane in the reciprocal space at three energy transfer values, $\hbar \omega=27$, 29 and $31 \mathrm{meV}$, in the AFM phase $(T=18 \mathrm{~K})$. The wavevector coordinates are given in reciprocal lattice units (r.l.u.), defined as 1 r.l.u. $=4 \pi / \sqrt{3} a$ for the $(H \bar{H} 0)$ direction and 1 r.l.u. $=2 \pi / c$ for the $(00 L)$ direction, where $a=9.079 \AA$ and $c=10.38 \AA$ are the lattice parameters in the hexagonal setting. ${ }^{44}$ The tail of the direct neutron beam, centered at $|\mathbf{Q}|=0$, and the $\mathrm{Al}$ powder lines originating from the sample holder have been subtracted from the data. At $\hbar \omega=27,29$ and $31 \mathrm{meV}$ [Fig. $1(\mathrm{a}-\mathrm{c})$ ], 1D intensity streaks orthogonal 

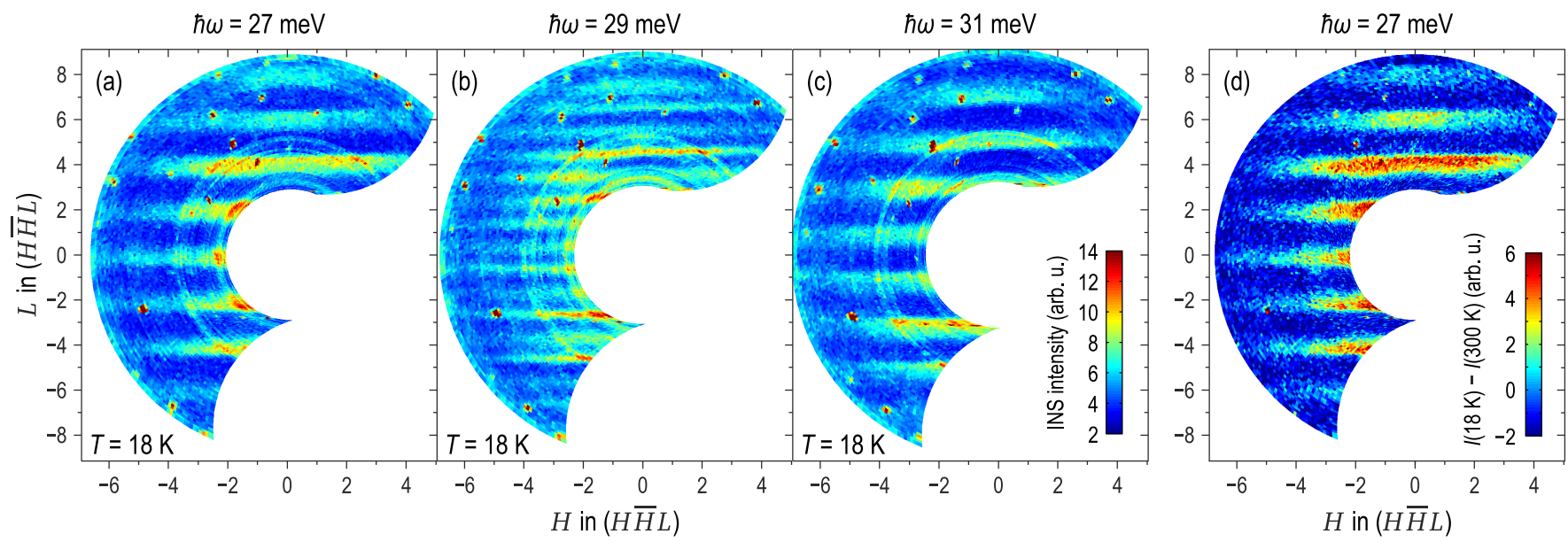

Fig. 1 (color online). (a)-(c) Constant-energy INS maps along the $(H \bar{H} L)$ plane in reciprocal space, measured in the AFM state $(T=18 \mathrm{~K})$ at three different energies indicated above each panel. The tail of the direct neutron beam and the Al powder lines have been subtracted from the data. The sharp spurious intensity spots originate from accidental Bragg intensity scattered incoherently by the analyzer and should be neglected. (d) Intensity difference between $T=18 \mathrm{~K}$ and $T=300 \mathrm{~K}$ data at $\hbar \omega=27 \mathrm{meV}$, shown to demonstrate the clean background-free magnetic signal and its form-factor decay with increasing $|\mathbf{Q}|$.

to the chain direction are observed at even, half-integer, and odd $L$ values, respectively, corresponding to the bottom, middle, and top of a dispersive magnon branch. The observed decrease in their intensity towards higher momentum transfer, $|\mathbf{Q}|$, is consistent with the $\mathrm{Co}^{3+}$ magnetic form factor, thereby confirming the magnetic origin of these excitations. To illustrate this more clearly, in Fig. 1 (d) we additionally show an intensity difference map $[I(18 \mathrm{~K})-I(300 \mathrm{~K})]$ for $\hbar \omega=27 \mathrm{meV}$, where the intensity measured at $T=300 \mathrm{~K}$ (which is featureless in $\mathbf{Q}$ ) has been used as a background. Here the intensity streaks are more pronounced in the absence of the background contamination, showing an anisotropic form-factor decay of intensity towards higher $|\mathbf{Q}|$. The absence of any notable dispersion along the $(H \bar{H} 0)$ direction in Fig. 1 could originate from very small interchain interactions and/or their effective cancelation due to the geometric frustration. Assuming that a weak dispersion in the $(H \bar{H} 0)$ direction with a bandwidth smaller than half of the experimental resolution would be unobservable, we can put an upper bound of $\sim 0.5 \mathrm{meV}$ on the dispersion perpendicular to the chains.

To study the magnon dispersion along the chains, we performed energy scans around $\mathbf{Q}=(00 L)$ at various temperatures, both above and below $T_{\mathrm{N}}$. In the Flatcone configuration, every energy scan follows a curved 2D surface in the momentum-energy space. However, since the magnetic signal does not depend on the momentum component orthogonal to the chains, in Fig. 2 we present the data in the two-dimensional $(L, \hbar \omega)$ projection, neglecting the $H$ component of the momentum without any loss of information. Three representative spectra at $T=1.5,50$ and $109 \mathrm{~K}$ are shown in Fig. $2(\mathrm{a}-\mathrm{c})$. Additional temperature frames are also presented in the supplemental material (SM) ${ }^{40}$ Our main result illustrated by these figures is the observation of a dispersive narrow magnon band with a large spin gap, which "melts" gradually upon warming. The average magnon energy, $E_{0}$, and its $T$-dependence are consistent with the location of an inelastic peak in earlier INS data measured on a powder sample. ${ }^{45}$ The dispersion reaches its minimum at the FM zone center (even $L$ ), with a spin gap of $\sim 27 \mathrm{meV}$ at $1.5 \mathrm{~K}$, and its maximum at the zone boundary (odd $L$ ) near $\sim 30.5 \mathrm{meV}$, thereby explaining the location of the intensity streaks in Fig. 1. The bandwidth of the magnon branch along the $\mathbf{c}^{*}$-direction therefore amounts to $\sim 3.5 \mathrm{meV}$. The large ratio of the spin gap to the bandwidth $(\sim 8)$ and the absence of dispersion perpendicular to the chains indicate the highly $1 \mathrm{D}$ nature of this material in spin space and in real space, respectively. Above $T_{\mathrm{N}}$, the gap in the magnetic excitation spectrum starts to fill gradually, yet the magnon band persists at least up to $150 \mathrm{~K}$ [see Fig. S1 (b, c) in the SM].

To analyze the magnon dispersion along the chains, we used an effective spin Hamiltonian that includes both the exchange and single-ion anisotropy terms:

$$
H=-\sum_{i}\left[J_{z} S_{i}^{z} S_{i+1}^{z}+J_{x y}\left(S_{i}^{x} S_{i+1}^{x}+S_{i}^{y} S_{i+1}^{y}\right)+D\left(S_{i}^{z}\right)^{2}\right]
$$

Here $i$ is the site index for the $\mathrm{Co}_{\mathrm{TP}}^{3+}$ ions in the chain, and the $z$-axis is directed along the chains. The first and second terms in Eq. 1 describe the coupling between longitudinal $\left(J_{z}\right)$ and transverse $\left(J_{x y}\right)$ components of neighboring $\mathrm{Co}_{\mathrm{TP}}^{3+}$ spins, respectively, and $D$ is the single-ion anisotropy for the $\mathrm{Co}_{\mathrm{TP}}^{3+}$ ions. The AFM interchain interactions were excluded from Eq. 1 for simplicity, as they are very weak and thus do not affect the dispersion along the chains significantly.

Due to the high value of the $\mathrm{Co}_{\mathrm{TP}}^{3+}$ spins, magnetic excitations with different spin quantum numbers $(|\Delta S| \leq 2 S)$ are possible in the system. For instance, the simplest excited state of an individual FM chain (neglecting the interchain interactions) is realized when a single spin flips, creating two domain walls, which corresponds to a magnetic excitation with $|\Delta S|=4$ whose energy is independent of $D$. Although all of these excitations may contribute to thermodynamic properties, the selection rule dictated by the neutron spin restricts the excitations observable by INS to only those with $\Delta S= \pm 1$. Within the framework of the conventional linear spin-wave theory, their dispersion is given by

$$
\hbar \omega_{L}=2 S\left[\left(D+J_{z}\right)-J_{x y} \cos (\pi L)\right] .
$$

By fitting the experimental data to this model, as described in the SM ${ }^{40}$ we obtained the magnon dispersion for all measured temperatures, shown in Fig. 3 (a). At $T=1.5 \mathrm{~K}$, the following parameter values resulted from our fitting procedure: $D+J_{z}=7.20 \pm 0.02 \mathrm{meV}$ and $J_{x y}=0.42 \pm 0.01 \mathrm{meV}$ 


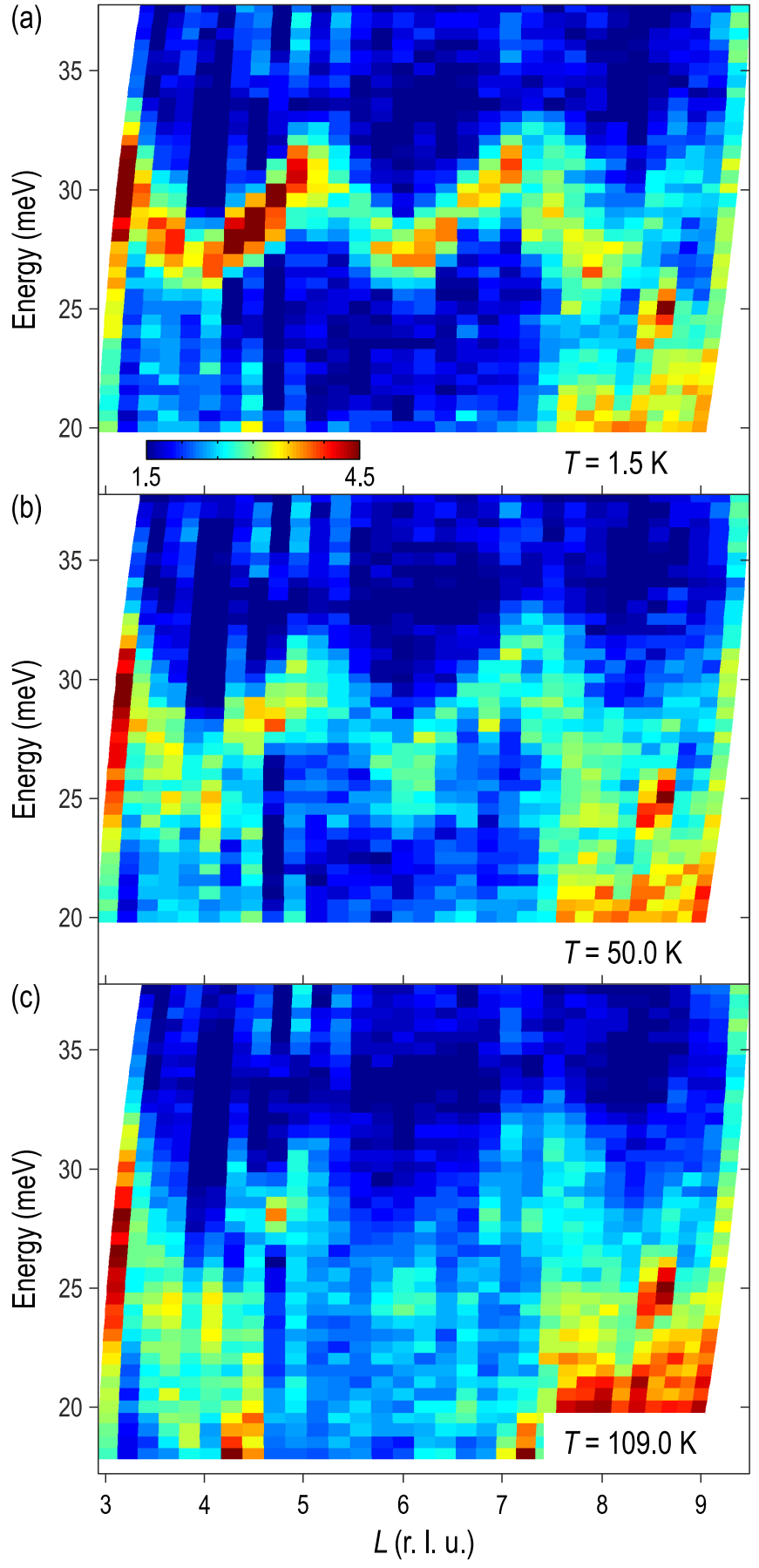

Fig. 2 (color online). Intensity color maps of the observed inelastic neutron spectrum at (a) $1.5 \mathrm{~K}$, (b) $50 \mathrm{~K}$, and (c) $109 \mathrm{~K}$. The data are projected onto the $(00 \mathrm{~L})$ direction of the momentum. The $T$ independent contributions from the tail of the direct beam at small $|\mathrm{Q}|$ and $\mathrm{Al}$ powder lines from the sample holder around $L=4.5$ have been subtracted from the data.

(assuming $S=2$ ). We note that $J_{z}$ and $D$ enter Eq. (2) additively and therefore cannot be extracted separately from the magnon dispersion alone. Fig. 3 (b) also shows the $T$ dependence of the main fitting parameters: the overall peak intensity and the center of the magnon band, $E_{0}$. The observation of well defined magnetic excitations throughout the Brillouin zone up to at least $150 \mathrm{~K}$ suggests that FM correlations along the chains develop prior to the onset of the 3D magnetic LRO at $T \gg T_{\mathrm{N}}$. The intensity of the signal, the magnon energy and the spin gap remain essentially constant below $T_{\mathrm{N}}$, but start decreasing upon warming above
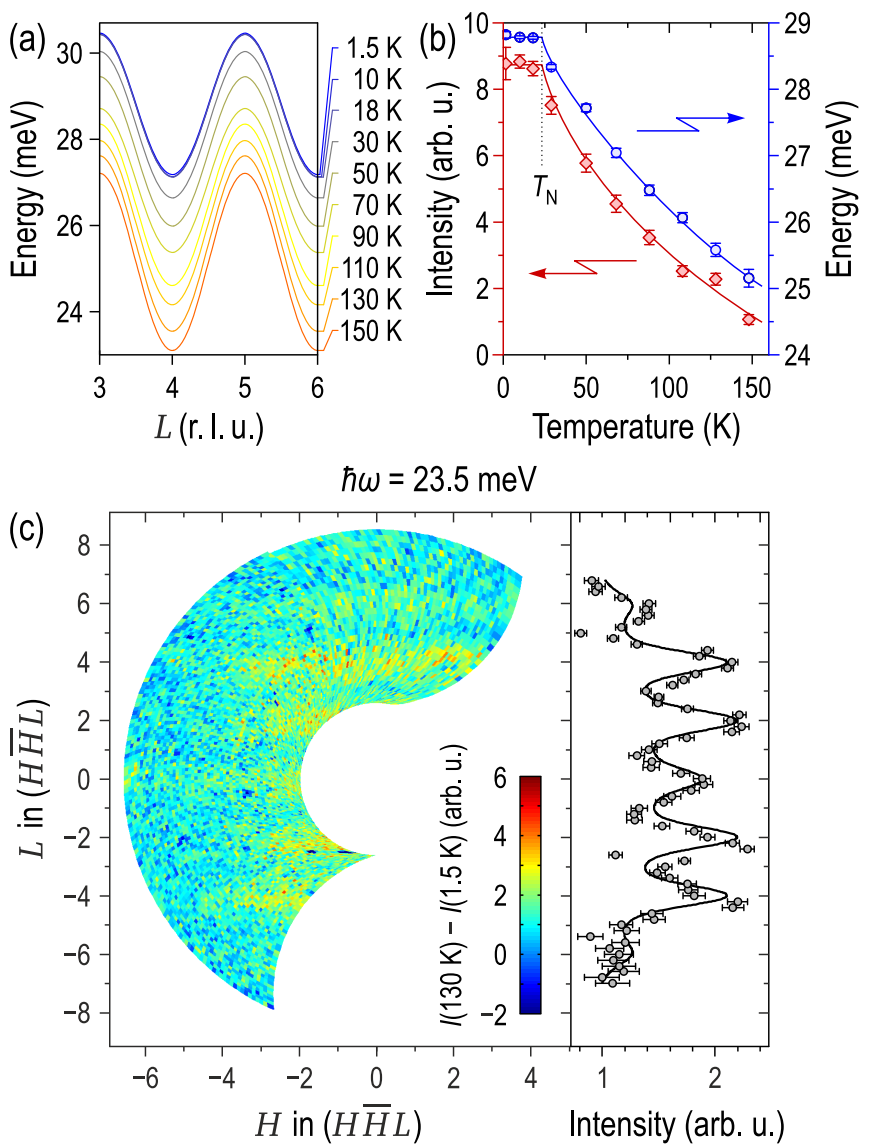

Fig. 3 (color online). (a) Fitted magnon dispersion curves along the chains at different temperatures, as indicated in the legend. Background substraction at every temperature was carried out in an iterative manner. ${ }^{40}$ (b) The overall peak intensity (diamond symbols) and the energy, $E_{0}$, corresponding to the center of the magnon dispersion curves (circles) obtained from the fit shown in panel (a). (c) Reciprocal space map at $\hbar \omega=23.5 \mathrm{meV}$ after subtraction of the background measured inside the spin gap at $T=1.5 \mathrm{~K}$, shown to demonstrate the transfer of spectral weight to lower energies upon warming. The projection of intensity onto the $L$ axis is shown to the right.

$T_{\mathrm{N}}$, while the bandwidth remains nearly $T$-independent. At $T \geq 200 \mathrm{~K}$, the signal broadens and is reduced in intensity below the statistical noise level in our data.

To demonstrate the softening of the magnon branch upon warming directly, without relying on the model fits, in Fig. 3 (c) we show the intensity difference, I $(130 \mathrm{~K})-$ $I(1.5 \mathrm{~K})$, taken at $\hbar \omega=23.5 \mathrm{meV}$. At $T=1.5 \mathrm{~K}$, this energy lies deep inside the spin gap, as one can see from Fig. 2 (a), and therefore the base-temperature data serve here as a measure of background to obtain the clean magnetic signal at $T=130 \mathrm{~K}$. The observed intensity streaks in Fig. 3 (c) confirm the shift of the magnetic spectral weight from higher energies down into the spin-gap region, which can be attributed to the reduction of the intrachain FM correlations by thermal excitations.

In Figs. 4 (a, b), we examine the spectral-weight redistribution in more detail by following the $T$-dependence of the dynamic spin susceptibility, $\chi_{T}^{\prime \prime}(\omega)$, momentum-integrated within \pm 0.25 r.l.u. around the bottom $(L=6)$ and top $(L=5)$ of the magnon band, respectively. The high-temperature background observed at $300 \mathrm{~K}$ has been subtracted to emphasize the changes in the magnetic signal with respect to ambient temperature. At $1.5 \mathrm{~K}$, sharp peaks can be seen 


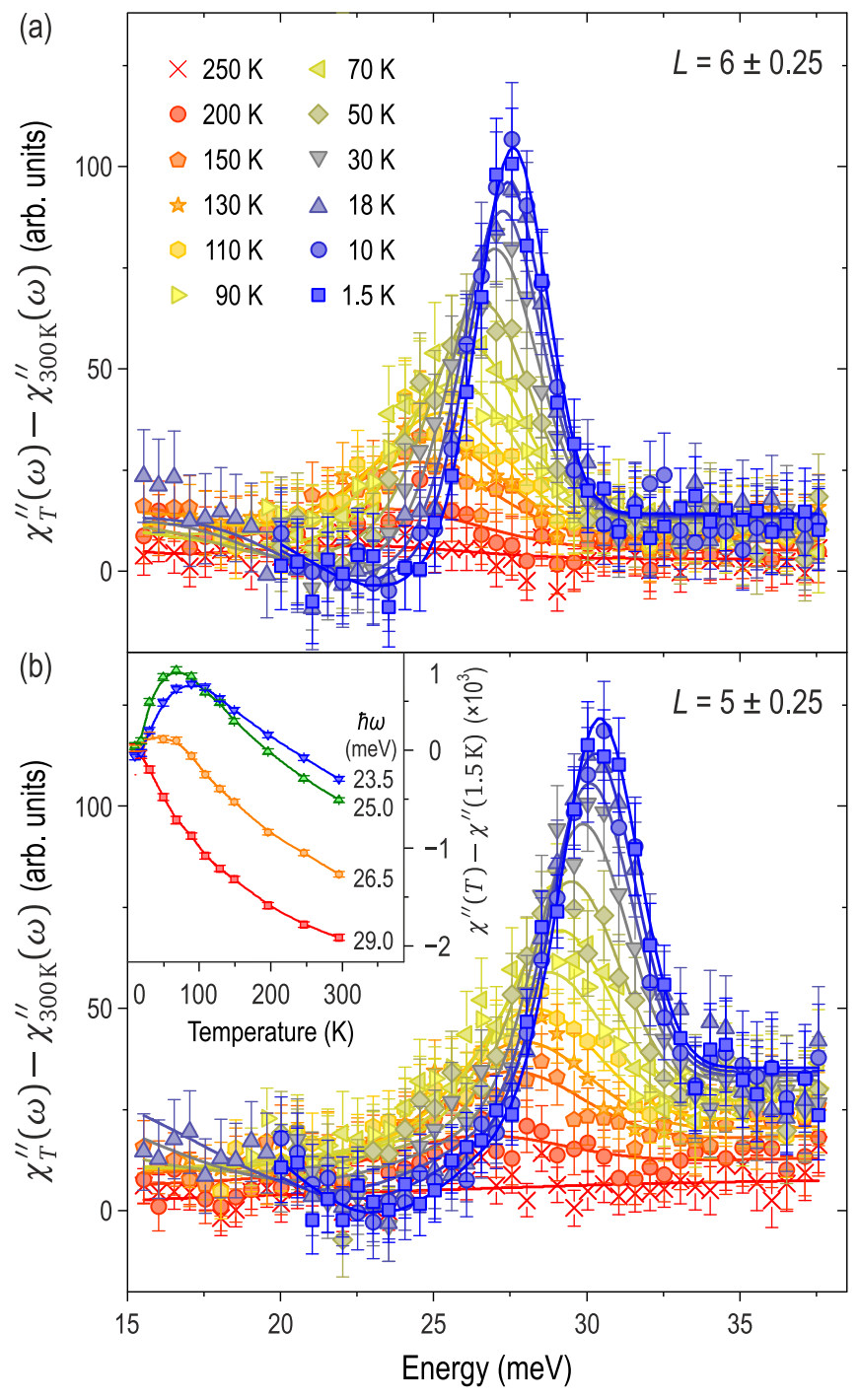

Fig. 4 (color online). Temperature variation of $\chi_{T}^{\prime \prime}(\omega)$, integrated over the wave vector range \pm 0.25 r.l.u. around (a) $L=5$ and (b) $L=6$. The background observed at $300 \mathrm{~K}$ was subtracted from the data. Solid lines are empirical fits to the data. The inset shows $T$-dependent changes in the full Q-integrated INS intensity with respect to its low- $T$ values, averaged over $\pm 0.5 \mathrm{meV}$ around $\hbar \omega=23.5,25,26.5$, and $29 \mathrm{meV}$. These data were obtained by $\mathbf{Q}-$ and $\omega$-integration from energy scans such as those shown in Fig. 2.

near 27.5 and $30.5 \mathrm{meV}$ for even and odd $L$, respectively, in agreement with the fitted magnon dispersion. These peaks gradually broaden and shift to lower energies upon warming, illustrating the softening of the magnon mode and the reduction of the spin gap. In the inset to Fig. 4 (b), we also present the change in the Q-integrated $\chi_{T}^{\prime \prime}(\omega)$ within several energy windows, averaged over $\pm 0.5 \mathrm{meV}$ around $\hbar \omega=23.5,25,26.5$, and $29 \mathrm{meV}$, with respect to its low- $T$ values. The intensity at low energies experiences an initial enhancement due to the reduction of the spin gap, while the intensity within the magnon band $(29 \mathrm{meV})$ is reduced.

We conclude by comparing our results with earlier complementary measurements. A spin gap of $\sim 100 \mathrm{~K}(8.6 \mathrm{meV})$ was previously estimated from ${ }^{59}$ Co spin-lattice relaxation rates. ${ }^{46}$ Intrachain $\mathrm{FM}$ correlations above $T_{\mathrm{N}}$ have also been reported from the Mössbauer effect (in Eu-doped $\left.\mathrm{Ca}_{3} \mathrm{Co}_{2} \mathrm{O}_{6}\right),{ }^{47}$ zero-field muon-spin relaxation ${ }^{48}$ and Ramanscattering ${ }^{49}$ measurements. These correlations are dynamic in nature and do not result in any enhancement of intensity at the nuclear Bragg positions, according to numerous neutron-diffraction studies. ${ }^{15,21,33,34,37}$ Our measured value of the spin gap in $\mathrm{Ca}_{3} \mathrm{Co}_{2} \mathrm{O}_{6}$ is similar to the one determined recently for the $1 \mathrm{D}$ copper-iridium oxide $\mathrm{Sr}_{3} \mathrm{CuIrO}_{6}$ using resonant inelastic x-ray scattering, ${ }^{52}$ where it was attributed to an Ising-like exchange anisotropy, while the band width in the latter compound is approximately 6 times larger than in $\mathrm{Ca}_{3} \mathrm{Co}_{2} \mathrm{O}_{6}$. It is appropriate to note that the presence of magnetic excitations above $T_{\mathrm{N}}$ were also reported in other FM spin-chain compounds, such as $\mathrm{CsNiF}_{3}$ (Ref. 50) or $\mathrm{CoCl}_{2} \cdot 2 \mathrm{D}_{2} \mathrm{O}$ (Ref. 51). However, the persistence of the magnons up to temperatures as high as $\sim 6 T_{\mathrm{N}}$ has not been observed so far in any material, to our knowledge.

The implications of our results can also be considered in the context of a broader class of $1 \mathrm{D}$ cobalt chain systems, such as mono-atomic nanowires ${ }^{53}$ or single-chain magnets. ${ }^{54,55}$ As these systems are highly anisotropic in spin space and purely 1D in real space, they cannot exhibit any LRO at nonzero temperature. Still, for Co-nanowires, extremely strong FM correlations persist above the Curie temperature $(T=0)$, and the corresponding relaxation times are long enough to be studied by scanning tunneling spectroscopy and XMCD. ${ }^{53}$ For single-chain magnets, the relaxation of the magnetization has been observed in the ac susceptibility. ${ }^{54,55}$ Yet, these techniques do not yield quantitative information about the microscopic magnetic interactions, which we were able to extract here from neutronspectroscopy data on a bulk analogue.

In summary, we have observed a dispersing magnetic excitation in $\mathrm{Ca}_{3} \mathrm{Co}_{2} \mathrm{O}_{6}$, whose quasi-1D character evidences the prevalence of FM intrachain over AFM interchain interactions. The magnon dispersion along the chains, exhibiting an anisotropy gap 8 times larger than the bandwidth, allowed us to give a direct estimate of the anisotropy and exchange parameters in this system, which proves the strongly anisotropic Ising-type character of its magnetic moments.

We thank D. T. Adroja, C. D. Batista, G. Khaliullin, C. Mazzoli, and A. Zheludev for stimulating discussions. P. Y. P. acknowledges financial support by DFG within the Graduiertenkolleg GRK 1621. H. J. was supported by the Max Planck POSTECH Center for Complex Phase Materials with KR2011-0031558.

\footnotetext{
* Corresponding author: ajain@barc.gov.in

$\dagger$ Corresponding author: Dmytro.Inosov@tu-dresden.de

${ }^{1}$ R. Moessner and A. P. Ramirez, Phys. Today 59, 24 (2006).

${ }^{2}$ L. Balents, Nature (London) 464, 199 (2010).

${ }^{3}$ C. Lacroix, P. Mendels and F. Mila (Eds.), Introduction to Frustrated Magnetism (Springer, 2011).

${ }^{4}$ M. Mekata, J. Phys. Soc. Jpn. 42, 76 (1977).
}

${ }^{5}$ M. F. Collins and O. Petrenko, Can. J. Phys. 75, 605 (1997).

${ }^{6}$ S. Nakatsuji et al., Science 309, 1697 (2005).

7 A. Olariu et al., Phys. Rev. Lett. 97, 167203 (2006).

8 S. Toth et al., Phys. Rev. Lett. 109, 127203 (2012).

9 B. Bernu, C. Lhuillier and L. Pierre, Phys. Rev. Lett. 69, 2590 (1992), and references therein.

10 G. H. Wannier, Phys. Rev. 79, 357 (1950).

11 R. Coldea et al., Science 327, 177 (2010). 
12 S. B. Lee, R. K. Kaul and L. Balents, Nature Phys. 6, 702 (2010).

13 S. A. J. Kimber et al., Phys. Rev. B 84, 104425 (2011).

14 W. B. Yelon, D. E. Cox and M. Eibschütz, Phys. Rev. B 12, 5007 (1975).

15 H. Kageyama, K. Yoshimura, K. Kosuge, H. Mitamura and T. Goto, J. Phys. Soc. Jpn. 66, 1607 (1997).

${ }^{16}$ V. Hardy et al., Phys. Rev. B 70, 064424 (2004); ibid. 70, 214439 (2004).

17 A. Maignan et al., J. Mater. Chem. 14, 1231 (2004).

18 C. L. Fleck, M. R. Lees, S. Agrestini, G. J. McIntyre and O. A. Petrenko, EPL 90, 67006 (2010).

19 P. J. Baker, J. S. Lord and D. Prabhakaran, J. Phys.: Condens. Matter 23, 306001 (2011).

20 T. Moyoshi and K. Motoya, J. Phys. Soc. Jpn. 80, 034701 (2011).

${ }^{21}$ S. Agrestini et al., Phys. Rev. Lett. 106, 197204 (2011).

22 N. Bellido, C. Simon and A. Maignan, Phys. Rev. B 77, 054430 (2008).

${ }^{23}$ Y. Shimizu, M. Horibe, H. Nanba, T. Takami and M. Itoh, Phys. Rev. B 82, 094430 (2010).

${ }^{24}$ A. Jain, S. Singh and S. M. Yusuf, Phys. Rev. B 74, 174419 (2006).

${ }^{25}$ V. Hardy, D. Flahaut, R. Frésard and A. Maignan, J. Phys. Condens. Matter 19, 145229 (2007).

${ }^{26}$ K. Takubo et al., Phys. Rev. B 71, 073406 (2005).

27 J.-G. Cheng, J.-S. Zhou and J. B. Goodenough, Phys. Rev. B 79, 184414 (2009).

28 T. Wei et al., Sci. Rep. 3, 1125 (2013).

29 T. Burnus et al., Phys. Rev. B 74, 245111 (2006).

${ }^{30}$ R. Frésard, C. Laschinger, T. Kopp and V. Eyert, Phys. Rev. B 69, 140405(R) (2004).

${ }^{31}$ Y. B. Kudasov, Phys. Rev. Lett. 96, 027212 (2006).

${ }^{32}$ X. Y. Yao, S. Dong and J.-M. Liu, Phys. Rev. B 73, 212415 (2006).

${ }^{33}$ S. Aasland, H. Fjellvåg and B. Hauback, Solid State Commun. 101, 187 (1997).

${ }^{34}$ H. Kageyama, K. Yoshimura, K. Kosuge, X. Xu and S. Kawano, J. Phys. Soc. Jpn. 67, 357 (1998).

35 O. A. Petrenko, Eur. Phys. J. B 47, 79 (2005).

36 A. Bombardi, C. Mazzoli, S. Agrestini and M. R. Lees, Phys. Rev. B 78, 100406(R) (2008).

37 S. Agrestini, L. C. Chapon, A. Daoud-Aladine, J. Schefer, A. Gukasov, C. Mazzoli, M. R. Lees and O. A. Petrenko, Phys. Rev. Lett. 101, 097207 (2008).
38 L. C. Chapon, Phys. Rev. B 80, 172405 (2009).

39 Y. Kamiya and C. D. Batista, Phys. Rev. Lett. 109, 067204 (2012).

40 See EPAPS Document No. [to be inserted by publisher] for the animated temperature dependence of the magnon dispersion with additional temperature frames (Fig. S1) and the results of sample characterization (Fig. S2).

41 A. Maignan et al., Eur. Phys. J. B 15, 657 (2000).

${ }^{42}$ V. Hardy, S. Lambert, M. R. Lees and D. McK. Paul, Phys. Rev. B 68, 014424 (2003).

43 The Flatcone configuration enables a convenient mapping of the reciprocal space at several constant energies. The final neutron wave vector was fixed to $k_{\mathrm{f}}=3 \AA^{-1}$, and $\mathrm{Si}(111)$ was used both for the monochromator and analyzer to reduce the contamination from higher-order neutrons.

${ }^{44}$ H. Fjellvåg et al., J. Sol. Stat. Chem. 124, 190 (1996).

45 S. Crowe, S. Agrestini, D. T. Adroja, M. Lees and D. Paul, Magnetic excitations in one-dimensional spin-chain systems: $\mathrm{Ca}_{3} \mathrm{CoMO}_{6}(\mathrm{M}=\mathrm{Mn}$ and Ir), ISIS Exp. Report \#510410 (Rutherford Appleton Laboratory, 2005), unpublished.

46 G. Allodi, R. De Renzi, S. Agrestini, C. Mazzoli and M. R. Lees, Phys. Rev. B 83, 104408 (2011).

47 P. L. Paulose, N. Mohapatra and E. V. Sampathkumaran, Phys. Rev. B 77, 172403 (2008).

48 S. Takeshita, J. Arai, T. Goko, K. Nishiyama and K. Nagamine, J. Phys. Soc. Jpn. 75, 034712 (2006).

49 S. Gohil, K. K. Iyer, P. Aswathi, S. Ghosh and E. V. Sampathkumaran, J. Appl. Phys. 108, 103517 (2010).

50 M. Steiner and B. Dorner, Solid State Commun. 12, 537 (1973).

51 J. K. Kjems, J. Als-Nielsen and H. Fogedby, Phys. Rev. B 12, 5190 (1975).

52 W.-G. Yin et al., Phys. Rev. Lett. 111, 057202 (2013).

${ }^{53}$ P. Gambardella, A. Dallmeyer, K. Maiti, M. C. Malagoli, W. Eberhardt, K. Kern and C. Carbone, Nature (London) 416, 301 (2002).

${ }^{54}$ L. Bogani, A. Caneschi, M. Fedi, D. Gatteschi, M. Massi, M. A. Novak, M. G. Pini, A. Rettori, R. Sessoli and A. Vindigni, Phys. Rev. Lett. 92, 207204 (2004).

55 A. Caneschi, D. Gatteschi, N. Lalioti, C. Sangregorio, R. Sessoli, G. Venturi, A. Vindigni, A. Rettori, M. G. Pini and M. A. Novak, Europhys. Lett. 58, 771 (2002). 


\section{Supplemental Material to the Letter}

\section{"One-Dimensional Dispersive Magnon Excitation in the Frustrated Spin-2 Chain System $\mathrm{Ca}_{3} \mathrm{Co}_{2} \mathrm{O}_{6}$ " by A. Jain et al.}

Here in Fig. S1 we present an animation showing the full set of INS data on $\mathrm{Ca}_{3} \mathrm{Co}_{2} \mathrm{O}_{6}$ as a function of temperature. The controls at the bottom of the figure allow to modify the frame rate, pause the animation, or browse through individual temperature frames. Panel (a) shows the raw INS data from energy scans after subtraction of $\mathrm{Al}$ powder lines, such as those presented in Fig. 2 in the paper. Panel (b) shows background-subtracted data. As the estimate for background in every frame, we used data from the next lower temperature, from which we subtracted the fitted model of the magnon dispersion. In the first iteration, we fitted the lowest-temperature data at $T=1.5 \mathrm{~K}$ with a two- dimensional model function that included the energy of the magnon, the amplitude of the dispersion, the peak width in energy (which was periodically modulated to account for the resolution effects), and the overall peak intensity as free fitting parameters. The model was multiplied by the $\mathrm{Co}^{3+}$ magnetic form factor to account for the decay of the signal with increasing $|\mathbf{Q}|$. After that, the result of the fit was subtracted from the data to get an estimate of background for the next temperature frame $(T=10 \mathrm{~K})$. This background was subtracted from the $T=10 \mathrm{~K}$ data, and the resulting dataset, shown in panel (b), was fitted to the same model to obtain the corresponding dispersion. (a) Raw INS data without $\mathrm{Al}$ powder lines

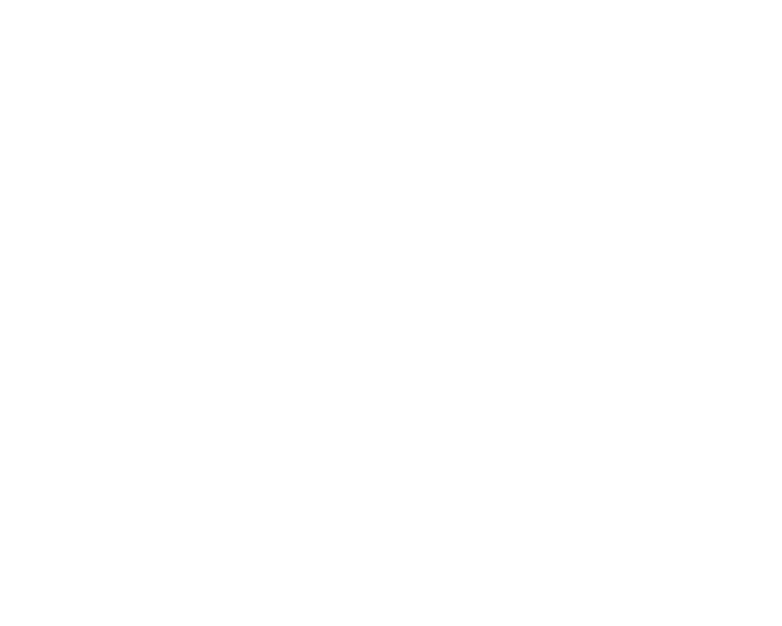

(c)

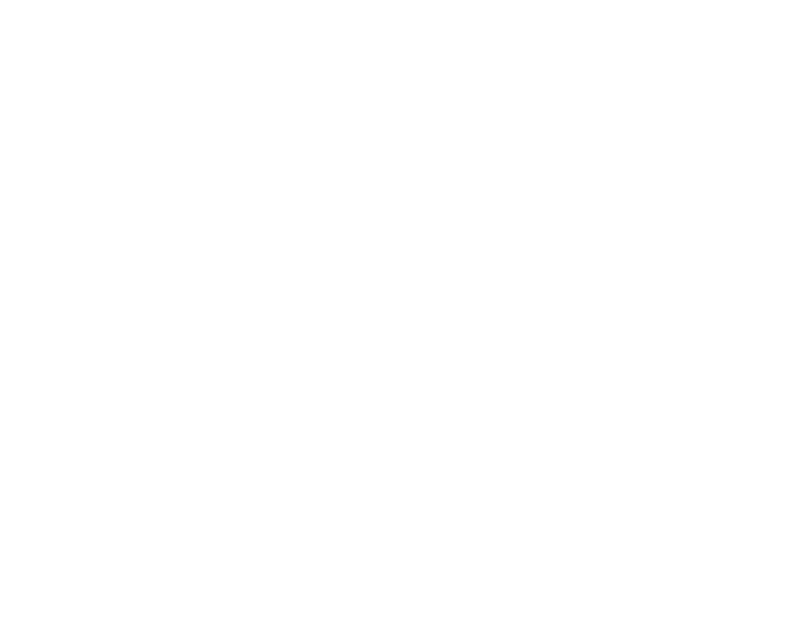

(b)

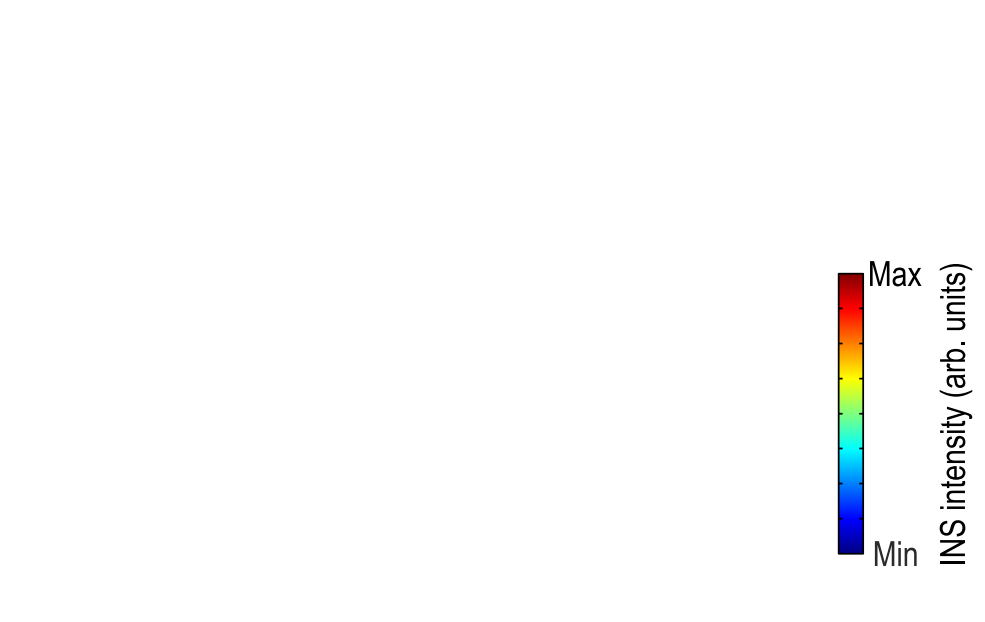

(d)

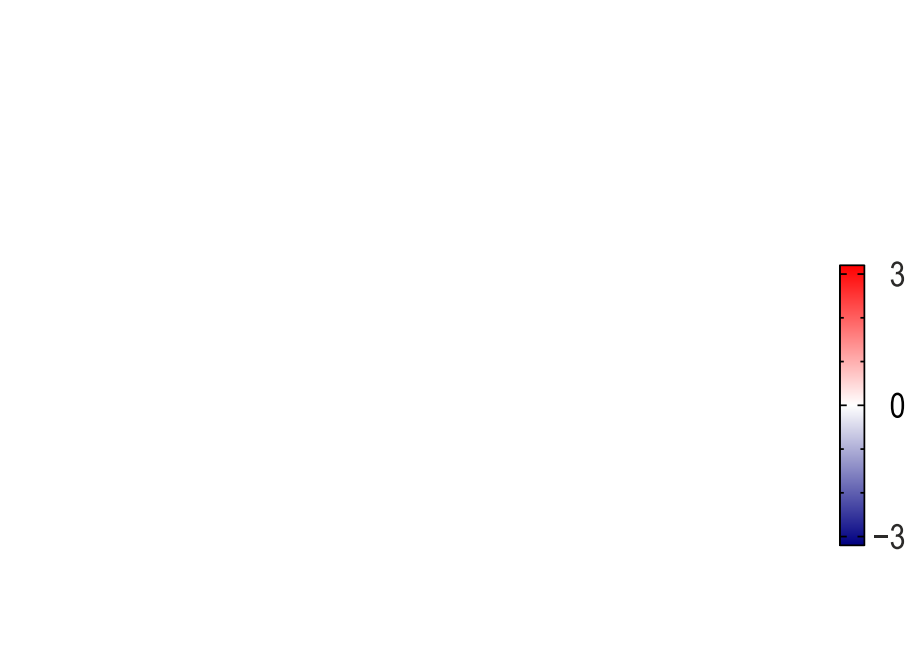

Fig. S1 (color online). Animations illustrating the temperature dependence of the magnon dispersion in $\mathrm{Ca}_{3} \mathrm{Co}_{2} \mathrm{O}_{6}$ along the ferromagnetic chain direction. (a) Raw INS data after subtraction of Al powder lines. (b) Background-subtracted data. Here, in every temperature frame we have subtracted the background contribution (data minus the fitted model) evaluated from the next lower temperature. (c) Temperature subtraction of the neutron scattering intensity, $I(T)-I(300 \mathrm{~K})$, that illustrates the transfer of magnetic spectral weight towards higher energies upon cooling. (d) Analogous subtraction for $I(T)-I(18 \mathrm{~K})$, illustrating the transfer of spectral weight to lower energies upon warming. 


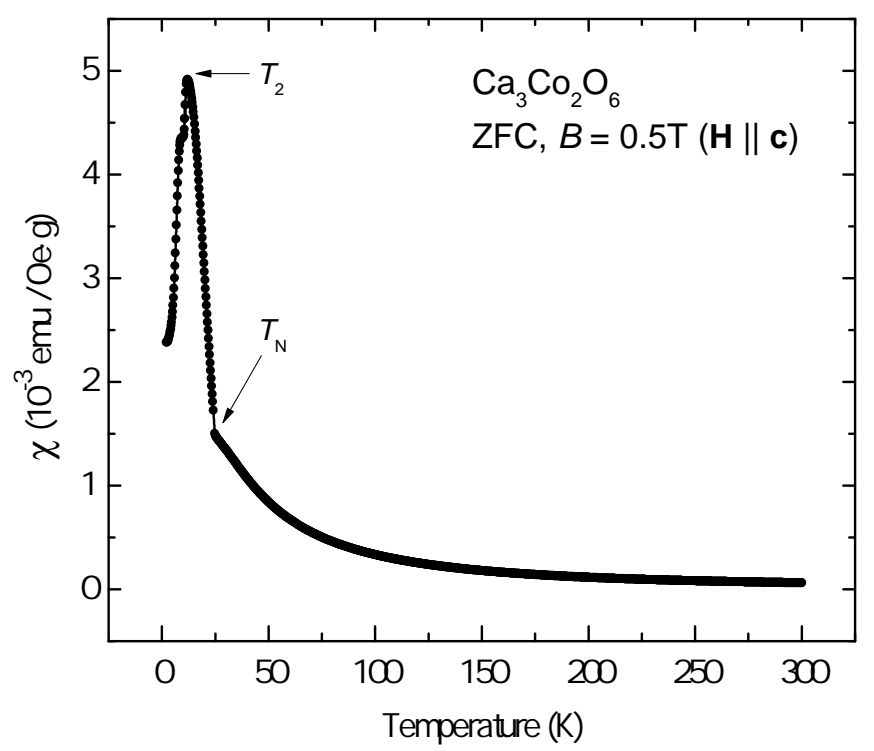

Fig. S2 (color online). Temperature dependence of the static magnetic susceptibility, measured after zero-field cooling in a constant magnetic field of $0.5 \mathrm{~T}$ applied parallel to the chain direction.

This model was then subtracted from the $T=10 \mathrm{~K}$ data to obtain the background for the next temperature frame $(T=$ $18 \mathrm{~K}$ ), and so on. Following this procedure, the dispersion at high temperatures, where the signal is masked by the uneven background, could be determined more reliably and up to higher temperatures than would be otherwise possible by fitting the raw data. The resulting dispersion curves and temperature-dependent parameters are plotted in Fig. 4 of the main paper.

Animations in Fig. S1 (c, d) show temperature subtractions of the raw data as an alternative way of eliminating the effects of the inhomogeneous background. In panel (c), we have subtracted the highest-temperature dataset $(T=$ $300 \mathrm{~K}$ ) from all frames to illustrate the formation of the magnon upon cooling, as the spectral weight from the spingap region is gradually transferred to higher energies, where the dispersive magnetic mode starts forming below $200 \mathrm{~K}$. In panel (d), we have similarly subtracted the $18 \mathrm{~K}$ dataset (immediately below $T_{\mathrm{N}}$ ) from all frames to illustrate the shift of the spectral weight towards lower energies while the mode is destroyed upon warming. In this representation of the data, one can clearly see the formation of a periodic intensity modulation within the spin-gap region (between 15 and $25 \mathrm{meV}$ ) at temperatures above $T_{\mathrm{N}}$ due to the gradual softening of the mode energy. The same structure can be also seen in the subtraction of constant-energy maps at $23 \mathrm{meV}$ that is shown in Fig. 3 (c) in the paper, as well as directly in the results of the fits presented in Figs. 3 (a) and (b).

In Fig. S2, we additionally show the results of a magnetic susceptibility measurement done by means of a SQUID magnetometer on one of the $\mathrm{Ca}_{3} \mathrm{Co}_{2} \mathrm{O}_{6}$ single crystals from the same batch as those used for the neutron mosaic sample. The field was applied parallel to the sample's c-axis after zero-field cooling (ZFC). The anomalies observed at $T_{\mathrm{N}}$ and at $T_{2} \approx 12 \mathrm{~K}$ agree with those reported previously on similar samples. $^{21,41,42}$ 